Donoso-Vázquez, T., Rubio Hurtado, M.J. y Vilà Baños, R. (2017). Las ciberagresiones en función del género. Revista de Investigación Educativa, 35(1), 197-214

DOI: http://dx.doi.org/10.6018/rie.35.1.249771

\title{
Las ciberagresiones en función del género'
}

\section{Cyberviolence \& gender-related indicators}

\author{
Trinidad Donoso-Vázquez*, María José Rubio Hurtado* y Ruth Vilà Baños** \\ * Profesora titular Universidad. Universidad Barcelona. \\ ** Profesora agregada interina. Universidad de Barcelona
}

\begin{abstract}
Resumen
El estudio analiza las experiencias en violencias de género en los entornos virtuales de chicos y chicas adolescentes e identifica factores asociados con el comportamiento ciberagresor en este colectivo. Participaron 155 estudiantes de primero y cuarto de ESO escolarizados en Barcelona, seleccionados mediante un muestreo por conveniencia. Se aplicó el Cuestionario de Violencias de Género 2.0. En relación a las experiencias, los resultados indican que todos los jóvenes de la muestra alguna vez han ejercido o han sido víctimas de agresiones, pero, sobre todo, han observado agresiones en los entornos virtuales. Las experiencias de agresiones se dan sobre todo en aspectos relacionados con los mitos del amor romántico, y con la homofobia.

En relación a los factores asociados al comportamiento ciberagresor, los resultados señalan que las agresiones en función del género correlacionan con agresiones de ciberbullying y los entornos virtuales más utilizados destacan como entornos de tipo más activo.

Palabras clave: ciberacoso; violencias de género; adolescencia; ciberagresores.
\end{abstract}

\begin{abstract}
The two main objectives of this study are: first, to get to know the experiences in adolescents about gender violence in virtual environments and; two, to identify factors associated with

Correspondencia: Trinidad Donoso-Vázquez. Profesora titular Universidad. Universidad Barcelona. E-mail: trinydonoso@ub.edu.

1 Se presentan algunos resultados parciales de la investigación “Violencias de género 2.0 - Convocatoria Fundación BBVA de Ayudas a proyectos de Investigación".
\end{abstract}


cyber-attackers' behaviour within the teenagers collective. 155 students of secondary education level participated. They were selected by convenience sampling. The following questionnaire was applied: Questionnaire Violences Gender 2.0. According to their experiences, results indicate that all adolescents in the sample have exercised aggression at any time, and all adolescents have been victims of aggression some time, but mainly they have observed aggression in virtual environments. The attacks are mainly related to the myths of romantic love, and to homophobia.

About the factors related to cyber-attackers' behaviour, the results indicate that gender attacks correlate with aggression in generating cyberviolence. Virtual environments are another factor associated with ciberaggression, highlighting the active virtual environments, like Skype or asken.

Keywords: cyberbullying; gender violence; adolescence; cyber-attackers.

\section{Introducción}

La violencia en espacios virtuales reproduce, mediante nuevos sistemas de producción y mantenimiento, jerarquías establecidas, donde asombrosamente el "cuerpo" no aparece de forma explícita, pero si el género. La identidad y roles de género predicen ciertas conductas offline que están tan socializadas y expandidas que se traspasan al espacio virtual (Estébanez \& Vázquez, 2013).

Aunque no de una manera completamente generalizada, pero sí bastante aceptada, el Cyberbullying se refiere al acoso en línea de los jóvenes y los adolescentes, sin que se medien necesariamente relaciones íntimas o de naturaleza sexual (Li, 2007). Acoso cibernético (Cyber Harassment) incluye actos tales como mensajes de acoso, amenazas, manipulación de la foto, la publicación de información personal, y la suplantación online. Para Calmaestra (2011) el término que comienza a utilizarse, por considerarse más amplio, es ciberagresión.

Entre la juventud, la intimidación offline es más frecuente que la ciberagresión (Defensor del Pueblo - UNICEF, 2006; Ortega, Calmaestra \& Mora-Merchán, 2008). En un estudio realizado en Córdoba (citado en Calmaestra, 2011) la cifra de prevalencia de cyberbullying es un 15,1\%, mientras que el de la intimidación offline es dos veces más alta $(32,7 \%)$. Aunque esta tendencia sigue manteniéndose (García Fernández, Romera, \& Ortega Ruiz, 2015), comienzan a aparecer signos claros de una inversión (Modecki, Minchin, Harbaugh, Guerra \& Runions, 2014).

En cuanto a la proporción de agresiones y victimizaciones, Calmaestra (2011) en el estudio realizado en Córdoba, encontró que la proporción de niños que participan como agresores es mayor que la proporción de niñas que participan como víctimas.

Resultados similares se encontraron en la Comunidad Valenciana (Buelga \& Pons, 2012), los chicos tienen puntuaciones estadísticamente más altas que las chicas en todas las conductas de hostigamiento. Las diferencias de género en el estudio de Beckman, Hagquist y Hellström (2013) eran mínimas, pero las chicas tenían más probabilidades de ser víctimas cibernéticas cuando se utiliza como punto de corte el acoso online ocasional.

Sin embargo, otros estudios se hacen eco de la simetría de las agresiones según el sexo. En un estudio de Alexy, Burgess, Baker y Smoyak (2005), con una muestra de universitarios y universitarias, los estudiantes tenían más probabilidad estadística 
que las mujeres de ser ciberacosados. Burke, Wallen, Vail-Smith y Knox (2011) obtuvieron resultados similares también en una muestra de universitarios. El $50 \%$ de los estudiantes encuestados (tanto hombres como mujeres) eran o el iniciador o víctima de esta conducta. Smith (2006) en una revisión de tres trabajos, encuentra diferencias estadísticamente significativas en uno de los estudios, las chicas más agredidas online, mientras que en otros dos estudios no se dan estas diferencias.

A tenor de todos los estudios revisados parece que no son concluyentes los resultados en cuanto a diferencias significativas por sexo en relación a agresiones y victimizaciones (Muñoz-Rivas, Graña Gómez, O’Leary \& González Lozano, 2007). A nuestro entender lo que se debe considerar, dentro de un enfoque de género, son las posibles consecuencias de las agresiones en hombres y en mujeres (Banyard \& Cross, 2008).

Los resultados sobre la prevalencia de la ciberagresión en jóvenes son muy variables. En la revisión de estudios realizada por Calvete, Orue, Estévez, Villardó y Padilla, (2010) los datos oscilan de 1,7\% a 35,7 \%. Estas diferencias podrían explicarse por los instrumentos de medida. Por ejemplo, en algunos de estos estudios el formato de los ítems es dicotómico, en otros una suma de incidencias y en otros se establece diferencia entre frecuencia del acoso -severo, medio, ocasional- y en otros no se establece esta diferencia. Los cuestionarios de medida más utilizados (Donoso-Vázquez, Rubio, Vilà, Velasco y Aneas, 2014) son de tipo escalar, validados en diferentes ocasiones y con diferentes muestras. Presentan una estructura factorial y un índice de consistencia interna normalmente bueno en sus diferentes aplicaciones. (Calvete et al., 2010; Hinduja \& Paching, 2008; Ortega, Calmaestra \& Mora-Merchán, 2008; Smith, 2006)

En cuanto a las teorías explicativas del fenómeno, los estudios realizados para la medición de la prevalencia, incidencia, extensión y características del ciberacoso han utilizado clasificaciones basadas en tipos de conductas exhibidas en la red o en las diferentes herramientas online utilizadas para ejercerlo. Una de las clasificaciones más utilizadas es la de Willard (citado en Calvete et al., 2010) que diferencia y analiza el maltrato en función de ocho modalidades de respuesta. En la misma línea, Martinez y Ortigosa (citado en Torres, 2013) confeccionan una lista de 13 tipos de prácticas de ciberacoso. Calmaestra (2011) recoge una relación de aquellos marcos explicativos que se han utilizado para interpretar las conductas de acoso, tanto offline como online. Estas teorías, sean del marco sociocultural, psicológicas o sistémicas, no consiguen ofrecer una visión específica e integral del acoso en función del género.

En este trabajo se adopta el marco heteronormativo patriarcal para analizar las agresiones contra las mujeres y contra homosexuales y transexuales, así como contra personas heterosexuales que se apartan de los cánones establecidos (Arisó \& Mérida, 2010; Finn \& Atkinson 2009; Southworth, Dawson, Fraser \& Tucker, 2005; SpenceDiehl, 2003). El patriarcado ejerce unas imposiciones sobre el género, establece cómo deben actuar las personas, comportarse y pensar según el género al que pertenecen. Cualquier cambio en estos patrones de comportamiento acarreará una presión social para restablecer el sistema normativo establecido. Cuando hablamos de violencia de género en internet nos referimos a la violencia que se ejerce contra las mujeres, por el hecho de tener una situación subalterna en el orden social y a la violencia que se ejerce contra otras personas por apartarse de los cánones establecidos para los géneros en 
el sistema patriarcal. La mayoría de las investigaciones existentes acerca del acoso en la red no analizan en profundidad la violencia de género que se da en ella. De igual manera, en las investigaciones sobre violencias de género no se incluye el análisis de las violencias que se pueden ejercer a través de la red. Existe, por lo general, una cantidad discreta de estudios destinados a estudiar específicamente la violencia de género en la red en comparación con la producción científica destinada a estudiar el acoso en general (Dimond, Fiesler \& Bruckman, 2011).

A esto hay que agregar la concepción restringida con que se aborda la violencia de género en Internet, únicamente desde la óptica de las relaciones afectivas, ya sea por parte de parejas actuales o por parte de exparejas cuando la relación ha terminado (Torres, 2013).

\section{Método}

\section{Objetivos}

El estudio presentado tuvo por objetivo general analizar las violencias de género 2.0 (VG2.0) en una muestra de adolescentes de Educación Secundaria Obligatoria.

De forma más específica, los objetivos planteados fueron:

- Conocer las experiencias de los y las adolescentes en VG2.0 (como ciberagresor/a, como cibervíctima y como ciberobservador/a).

- Identificar factores asociados con el comportamiento de ciberagresor/a de VG2.0 en el colectivo adolescente.

\section{Instrumento}

Se llevó a cabo un estudio por encuesta dirigido a adolescentes de Enseñanza Secundaria Obligatoria (ESO) de la provincia de Barcelona usando como instrumento de recogida de datos el cuestionario en modalidad on-line de Violencias de género 2.0 (Donoso-Vázquez, Rubio, Velasco \& Vilà, 2014).

El cuestionario examina la percepción que tienen las y los jóvenes sobre la violencia de género en los entornos virtuales, y recoge su perfil tecnológico y sus experiencias como agresores, víctimas y observadores de dicha violencia. Estas experiencias se han medido a través de tres escalas, cada una de las cuales considera y comparte 7 dimensiones relacionadas con la violencia derivada del sistema patriarcal: violencia por apartarse de la normatividad sexual femenina, violencia por transgredir la heteronormatividad sexual obligatoria, violencia debida a las imposiciones del canon de belleza heteronormativo, violencia ligada a estereotipos, violencia sexual, violencia por manifestar posiciones antipatriarcales, violencia asociada a mitos del amor romántico (véase tabla 1). Se incluyen finalmente algunos datos de caracterización sociodemográfica.

Las tres escalas del cuestionario muestran una fiabilidad alta con relación al índice de Alpha de Cronbach (tabla 1), superando el 0,8 en todos los casos, lo que indica una adecuada consistencia interna de sus ítems (Nunnally, 1978). 
La validez de contenido se estableció en base al juicio de personas expertas, las cuales fueron seleccionadas siguiendo los criterios de Skjong y Wentworht (2000), por su formación académica, su experiencia y su reconocimiento en la comunidad científica. Todos los ítems del instrumento obtuvieron al menos un $80 \%$ de acuerdo entre las 7 expertas con relación a su adecuación y validez para el objeto de estudio, un porcentaje considerado adecuado para que el ítem pertenezca al instrumento (Voutilainen \& Liukkonen, citado en Hyrkäs, Appelqvist-Schmidlechner, \& Oksa, 2003).

La validez de criterio se estableció mediante la escala de Experiencias como agresor, que correlaciona positivamente $(\mathrm{r}=0,638, \mathrm{p}=0,00)$ con la escala de Cyberbullying offending de Hinduja y Patchin (2010). Dicha escala está compuesta por 9 ítems orientados a conocer la agresión a través de Internet y el móvil obteniendo índices de fiabilidad buenos también, en torno al 0,8 de Alpha de Cronbach (Hinduja \& Patchin, 2008, 2010). Los ítems de esta escala son:

También se recogieron datos de esta escala en la muestra de participantes del estudio.

\section{Muestra}

En el estudio participaron 155 estudiantes de centros públicos de primero y cuarto de ESO (49,7\% y 50,3\% respectivamente) escolarizados en Barcelona, seleccionados mediante un muestreo por conveniencia. Las características relacionadas con el objeto de estudio que definen la muestra son las siguientes:

Un 51,4\% de chicas y un 48,6\% de chicos; el 28,39\% tiene una relación sentimental o de pareja y el 43,23\% considera que tiene alguna característica de vulnerabilidad para padecer violencia, como por ejemplo: su físico, su rendimiento académico, origen cultural, orientación sexual, etc.

Tabla 1

Estructura y fiabilidad del Cuestionario de violencias de género 2.0

\begin{tabular}{lll}
\hline \multicolumn{1}{c}{ Dimensiones } & \multicolumn{1}{c}{ Categorías de análisis } & \multicolumn{1}{c}{$\begin{array}{c}\text { Categorías de } \\
\text { análisis }\end{array}$} \\
\hline Contextualización & $\begin{array}{l}\text { Datos sociodemográficos } \\
\text { Percepción de tener características } \\
\text { personales de vulnerabilidad }\end{array}$ & \\
Perfil tecnológico y & Perfil de dominio y uso tecnológico \\
percepciones sobre la red & Percepción de internet como entorno violento & \\
& Percepción de impunidad de la red & \\
Experiencias de Violencia & Escala de ciberagresión de VG2.0 & \\
de Género 2.0 & Escala de cibervíctima de VG2.0 & 0,887 (23 ítems) \\
& Escala de ciberobservación de VG2.0 & 0,913 (23 ítems) \\
\hline
\end{tabular}

Elaboración propia 


\section{Procedimiento de recogida y análisis de datos}

El análisis de los resultados llevó a cabo mediante estadística descriptiva, aplicando contrastes de medias (t de Student) y pruebas de Chi cuadrado. Se aplicó un análisis de regresión lineal múltiple para identificar los factores asociados con las conductas de ciberagresión de VG 2.0 en el colectivo adolescente. También se aplicaron pruebas de colinealidad para garantizar la condición de no-colinealidad. Todos estos cálculos se realizaron con el paquete estadístico SPSS, versión 18.

\section{Resultados}

A continuación se presentan los resultados siguiendo el orden de las dimensiones que figuran en la tabla 1.

\section{Perfil tecnológico y percepciones sobre la red}

El perfil tecnológico se corresponde con un uso importante de la muestra en general de WhatsApp, Facebook y YouTube (77\%, 61\% y 74\% respectivamente). Diferenciando por sexo destaca Instagram en chicas (75\%) y Skype en chicos (72\%) (p=0,002, y 0,008, respectivamente). En menor medida usan Ask (23\%), Twitter (13\%) y Tuenti (3\%).

Mayoritariamente consideran que hay más violencia en los espacios online que offline, especialmente las chicas ( $86 \%$ respecto al $82 \%$ de los chicos). También perciben que existe impunidad en la red (el 73\% de las chicas y el 63\% de los chicos piensa que no se castiga a las personas que hacen algo malo en ella) y consideran que alguien como la policía, algún organismo especializado o la familia debería intervenir en los casos de violencia 2.0 (lo opina el $80 \%$ de las chicas y el $70 \%$ de los chicos).

Por último destaca que todos afirman poseer conocimientos relacionados con la seguridad en los entornos virtuales (sin que existan diferencias por sexo); concretamente saben bloquear a la gente que molesta (93\%), saben modificar las condiciones de privacidad en las redes sociales $(89,5 \%)$, saben denunciar las fotografías indebidas $(88,2 \%)$, y tiene antivirus el 69,3\%; en menor medida saben desactivar la geolocalización del móvil (60,8\%) y el 70\% no abre correos de desconocidos.

\section{Experiencias de violencias de género 2.0}

\section{Escala de ciberagresión}

Las ciberagresiones que ejercen con mayor frecuencia las y los participantes hacen referencia a conductas relacionadas con la violencia asociada a los mitos del amor romántico (ver tabla 2): el $27 \%$ dice que a veces controla la pareja, y el 3\% lo hace muchas veces. El 14\% dice que a veces inspecciona el móvil de la pareja, mientras que el 3\% lo hace muchas veces. En segundo lugar, también se ejerce violencia asociada a las imposiciones de belleza heteronormativas: el 17\% dice que a veces frecuenta páginas de puntuaciones por el físico de chicas y el 14\% dice que a veces insulta chicas por tener un físico poco atractivo (el 3\% dice que lo hacen muchas veces). 


\section{Tabla 2}

Porcentajes de respuestas obtenidas en las escalas sobre experiencias en VG 2.0 (ciberagresión, cibervíctima y ciberobservación) en cada una de sus dimensiones

\begin{tabular}{|c|c|c|c|c|c|c|c|c|c|}
\hline & \multicolumn{3}{|c|}{$\begin{array}{c}\text { Escala } \\
\text { ciberagresión }\end{array}$} & \multicolumn{3}{|c|}{$\begin{array}{c}\text { Escala } \\
\text { cibervictimización }\end{array}$} & \multicolumn{3}{|c|}{$\begin{array}{c}\text { Escala } \\
\text { ciberobservación }\end{array}$} \\
\hline & $\begin{array}{c}\text { ê } \\
\text { త্ } \\
\vdots \\
z\end{array}$ & 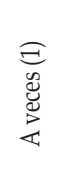 & 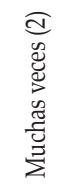 & 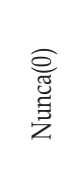 & 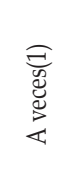 & 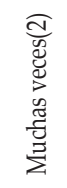 & $\underset{\substack{\tilde{U} \\
\Xi}}{\vdots}$ & 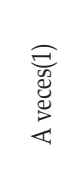 & 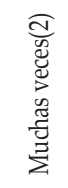 \\
\hline \multicolumn{10}{|c|}{ Violencia por apartarse de la normatividad sexual femenina } \\
\hline $\begin{array}{l}\text { Insultar por Internet a una chica } \\
\text { porque ha tenido diversas parejas }\end{array}$ & $86,5 \%$ & $13,5 \%$ &, $0 \%$ & $91,0 \%$ & $6,5 \%$ & $2,6 \%$ & $38,7 \%$ & $41,3 \%$ & $20,0 \%$ \\
\hline $\begin{array}{l}\text { Acosar una chica porque es } \\
\text { provocativa, con el móvil o redes } \\
\text { sociales }\end{array}$ & $85,8 \%$ & $13,5 \%$ & $6 \%$ & $93,5 \%$ & $5,8 \%$ & $6 \%$ & $51,6 \%$ & $33,5 \%$ & $14,8 \%$ \\
\hline $\begin{array}{l}\text { Meterse con una chica a través de } \\
\text { Internet porque no se interesa por } \\
\text { los chicos }\end{array}$ & $96,1 \%$ & $3,2 \%$ &, $6 \%$ & $96,8 \%$ & $3,2 \%$ &, $0 \%$ & $64,5 \%$ & $26,5 \%$ & $9,0 \%$ \\
\hline $\begin{array}{l}\text { Insultar en Internet a una chica } \\
\text { por no haber tenido relaciones } \\
\text { con chicos }\end{array}$ & $96,8 \%$ & $1,9 \%$ & $1,3 \%$ & $96,1 \%$ & $1,9 \%$ & $1,9 \%$ & $61,9 \%$ & $26,5 \%$ & $11,6 \%$ \\
\hline $\begin{array}{l}\text { Meterse con un chico por tener un } \\
\text { físico poco masculino }\end{array}$ & $90,3 \%$ & $7,7 \%$ & $1,9 \%$ & $94,2 \%$ & $4,5 \%$ & $1,3 \%$ & $54,8 \%$ & $31,6 \%$ & $13,5 \%$ \\
\hline \multicolumn{10}{|c|}{ Violencia por transgredir la heteronormatividad sexual obligatoria } \\
\hline $\begin{array}{l}\text { Difundir en Internet la orientación } \\
\text { sexual de alguien sin su permiso }\end{array}$ & $96,1 \%$ & $3,9 \%$ &, $0 \%$ & $96,1 \%$ & $3,2 \%$ & $6 \%$ & $62,6 \%$ & $29,7 \%$ & $7,7 \%$ \\
\hline $\begin{array}{l}\text { Suplantar la identidad de alguien } \\
\text { haciéndose pasar por homosexual } \\
\text { o transexual y ridiculizarlo en } \\
\text { Internet o móvil }\end{array}$ & $91,0 \%$ & $7,1 \%$ & $1,9 \%$ & $95,5 \%$ & $3,9 \%$ &, $6 \%$ & $56,1 \%$ & $33,5 \%$ & $10,3 \%$ \\
\hline $\begin{array}{l}\text { Insultar en la red a una } \\
\text { persona por ser o pensar que es } \\
\text { homosexual o transexual }\end{array}$ & $93,5 \%$ & $5,8 \%$ &, $6 \%$ & $96,1 \%$ & $2,6 \%$ & $1,3 \%$ & $62,6 \%$ & $23,2 \%$ & $14,2 \%$ \\
\hline
\end{tabular}

Violencia debida a las imposiciones del canon de belleza heteronormativo

\begin{tabular}{|c|c|c|c|c|c|c|c|c|c|}
\hline $\begin{array}{l}\text { Insultar a una chica por tener un } \\
\text { físico poco atractivo }\end{array}$ & $83,2 \%$ & $14,2 \%$ & $2,6 \%$ & $83,2 \%$ & $14,2 \%$ & $2,6 \%$ & $46,5 \%$ & $36,1 \%$ & $17,4 \%$ \\
\hline $\begin{array}{l}\text { Crear, participar o consultar una } \\
\text { página web que pone puntuación } \\
\text { al físico de las chicas }\end{array}$ & $81,9 \%$ & $17,4 \%$ & $6 \%$ & $89,7 \%$ & $9,0 \%$ & $1,3 \%$ & $59,4 \%$ & $25,2 \%$ & $15,5 \%$ \\
\hline $\begin{array}{l}\text { Mostrar la foto de una chica } \\
\text { simplemente como un objeto } \\
\text { sexual en redes sociales }\end{array}$ & $93,5 \%$ & $4,5 \%$ & $1,9 \%$ & $95,5 \%$ & $3,9 \%$ &, $6 \%$ & $56,8 \%$ & $31,0 \%$ & $12,3 \%$ \\
\hline \multicolumn{10}{|c|}{ Violencia basada en estereotipos } \\
\hline $\begin{array}{l}\text { Por manifestar opiniones te han } \\
\text { dicho "ves a fregar", o "a la cocina" }\end{array}$ & $85,8 \%$ & $12,3 \%$ & $1,9 \%$ & $85,2 \%$ & $12,3 \%$ & $2,6 \%$ & $58,7 \%$ & $27,1 \%$ & $14,2 \%$ \\
\hline \multicolumn{10}{|c|}{ Violencia sexual } \\
\hline $\begin{array}{l}\text { Amenazar una chica para } \\
\text { mantener una relación de pareja }\end{array}$ & $96,1 \%$ & $3,9 \%$ &, $0 \%$ & $91,6 \%$ & $6,5 \%$ & $1,9 \%$ & $64,5 \%$ & $25,2 \%$ & $10,3 \%$ \\
\hline
\end{tabular}




\begin{tabular}{|c|c|c|c|c|c|c|c|c|c|}
\hline $\begin{array}{l}\text { Llenar el correo de alguien con } \\
\text { contenido sexual }\end{array}$ & $94,8 \%$ & $3,9 \%$ & $1,3 \%$ & $94,8 \%$ & $4,5 \%$ & $6 \%$ & $76,8 \%$ & $17,4 \%$ & $5,8 \%$ \\
\hline $\begin{array}{l}\text { Conseguir fotos de una persona } \\
\text { para hacerle chantaje para } \\
\text { aprovecharse sexualmente de ella }\end{array}$ & $94,8 \%$ & $3,9 \%$ & $1,3 \%$ & $94,2 \%$ & $3,9 \%$ & $1,9 \%$ & $67,1 \%$ & $25,2 \%$ & $7,7 \%$ \\
\hline $\begin{array}{l}\text { Difundir vídeos / fotos sexis } \\
\text { de una chica en Internet sin su } \\
\text { permiso }\end{array}$ & $93,5 \%$ & $5,2 \%$ & $1,3 \%$ & $96,8 \%$ & $3,2 \%$ &, $0 \%$ & $56,1 \%$ & $31,0 \%$ & $12,9 \%$ \\
\hline \multicolumn{10}{|c|}{ Violencia por manifestar posiciones antipatriarcales } \\
\hline $\begin{array}{l}\text { Meterse con alguien en Internet } \\
\text { por su ideología feminista }\end{array}$ & $93,5 \%$ & $5,2 \%$ & $1,3 \%$ & $94,8 \%$ & $3,2 \%$ & $1,9 \%$ & $68,4 \%$ & $24,5 \%$ & $7,1 \%$ \\
\hline $\begin{array}{l}\text { meterse con alguien porque está a } \\
\text { favor del movimiento LGBTI }\end{array}$ & $96,8 \%$ & $2,6 \%$ & $6 \%$ & $96,1 \%$ & $6 \%$ & $3,2 \%$ & $63,9 \%$ & $25,2 \%$ & $11,0 \%$ \\
\hline \multicolumn{10}{|c|}{ Violencia asociada a mitos amor romántico } \\
\hline $\begin{array}{l}\text { Controlar la pareja en Facebook, } \\
\text { Twitter...] }\end{array}$ & $70,3 \%$ & $26,5 \%$ & $3,2 \%$ & $82,6 \%$ & $13,5 \%$ & $3,9 \%$ & $47,7 \%$ & $34,2 \%$ & $18,1 \%$ \\
\hline $\begin{array}{l}\text { Conocer la contraseña de la pareja } \\
\text { en las redes sociales para bloquear } \\
\text { amistades }\end{array}$ & $91,0 \%$ & $7,7 \%$ & $1,3 \%$ & $87,7 \%$ & $10,3 \%$ & $1,9 \%$ & $51,0 \%$ & $36,8 \%$ & $12,3 \%$ \\
\hline $\begin{array}{l}\text { Controlar la pareja por } \\
\text { geolocalización }\end{array}$ & $93,5 \%$ & $5,8 \%$ & $6 \%$ & $92,9 \%$ & $6,5 \%$ & $6 \%$ & $66,5 \%$ & $25,2 \%$ & $8,4 \%$ \\
\hline $\begin{array}{l}\text { Coger el móvil de la pareja para } \\
\text { ver llamadas e inspeccionar }\end{array}$ & $82,6 \%$ & $14,2 \%$ & $3,2 \%$ & $82,6 \%$ & $14,8 \%$ & $2,6 \%$ & $51,6 \%$ & $36,1 \%$ & $12,3 \%$ \\
\hline $\begin{array}{l}\text { Obligar a la pareja a quitar fotos } \\
\text { de amigos en FB o dejar de } \\
\text { whatsapear con alguien }\end{array}$ & $92,3 \%$ & $6,5 \%$ & $1,3 \%$ & $90,3 \%$ & $7,7 \%$ & $1,9 \%$ & $58,1 \%$ & $29,0 \%$ & $12,9 \%$ \\
\hline
\end{tabular}

\section{Escala de cibervictimización}

Como cibervíctimas, las conductas manifestadas son algo más frecuentes: el 15\% dice que a veces la pareja le inspecciona el móvil (al 3\% se lo hacen muchas veces); el $14 \%$ dice que está siendo a veces controlado por la pareja en las redes sociales (el $4 \%$ dice que muchas veces); $\mathrm{y}$ al $14 \%$ le insultan a veces por tener un físico poco atractivo (al 3\% le ha sucedido muchas veces).

\section{Escala de ciberobservación}

Finalmente se constata que las conductas en violencia de género 2.0 son mucho más observadas que protagonizadas e incluso se muestran tendencias diferentes a las anteriores. El $41 \%$ dice haber observado a veces que se insulta a una chica por tener varias parejas (el 20\% ha observado esta conducta muchas veces). El 37\% dice haber visto como algunos conocen las contraseñas de sus parejas para bloquear amistades (el 12\% lo ha visto muchas veces); y el 36\% ha observado cómo se inspeccionan móviles de las parejas (12\% muchas veces). El 36\% también ha observado como a veces se insultan chicas por tener un físico poco atractivo y el $17 \%$ lo ha visto muchas veces. Se observan algo más las conductas de VG 2.0 entre sus iguales (media de 38 sobre 69), y en menor medida se considera participar de ellas, como ciberagresores (media de 25 sobre 69) y como cibervíctimas (media de 26 sobre 69), como se aprecia en la tabla 3. 


\section{Contraste de las escalas por sexo}

Si contrastamos las puntuaciones de las tres escalas por sexo, observamos que existen diferencias en algunos aspectos:

- Las chicas observan más todas las conductas de VG 2.0 que los chicos (tabla 5), siendo estadísticamente significativa esta diferencia, excepto en la dimensión "transgredir la heteronormatividad sexual obligatoria (los actos de violencia relacionados con esta dimensión son observados por igual por chicos y chicas). Esta dimensión identifica las ciberagresiones a homosexuales, lesbianas y transexuales.

- En las dimensiones de la escala de experiencias en ciberagresión (tabla 3) puntúan más alto los chicos, pero esta diferencia sólo es estadísticamente significativa en las conductas asociadas a la "transgresión de la heteronormatividad sexual obligatoria", lo que significa que los chicos tienden a tener más conductas violentas online contra gais, lesbianas, transexuales, etc.

- En la escala de Experiencias como cibervíctimas (tabla 4), puntúan más alto las chicas, aunque la diferencia significativa se da en las conductas de violencia asociadas a mitos del amor romántico, lo que indica que las chicas padecen en mayor medida que los chicos este tipo de VG 2.0.

Tabla 3

Contraste por sexo en las puntuaciones de la escala ciberagresión en cada una de sus dimensiones

\begin{tabular}{|c|c|c|c|c|c|}
\hline Dimensiones de la escala & $\begin{array}{l}\text { Mínima } \\
\text { teórica }\end{array}$ & $\begin{array}{l}\text { Máxim. } \\
\text { teórica }\end{array}$ & $\begin{array}{c}\text { Chicas } \\
\text { Mean (SD) }\end{array}$ & $\begin{array}{c}\text { Chicos } \\
\text { Mean (SD) }\end{array}$ & $\begin{array}{c}t \\
(p)\end{array}$ \\
\hline $\begin{array}{l}\text { Apartarse de la normatividad } \\
\text { sexual femenina }\end{array}$ & 5 & 15 & $5,38(, 64)$ & $5,66(1,48)$ & $-1,40(, 163)$ \\
\hline $\begin{array}{l}\text { Transgredir heteronormatividad } \\
\text { sexual obligatoria }\end{array}$ & 3 & 9 & $3,11(, 46)$ & $3,34(, 73)$ & $-2,20(, 030)$ \\
\hline $\begin{array}{l}\text { Imposiciones del canon de } \\
\text { belleza heteronormativo }\end{array}$ & 3 & 9 & $3,45(, 84)$ & $3,46(, 94)$ & $-0,80(, 937)$ \\
\hline Estereotipos (rol familiar) & 1 & 3 & $1,21(, 44)$ & $1,13(, 42)$ & $1,04(, 300)$ \\
\hline Violencia sexual & 4 & 12 & $4,15(, 55)$ & $4,40(1,14)$ & $-1,61(, 111)$ \\
\hline $\begin{array}{l}\text { Violencia por manifestar } \\
\text { posiciones antipatriarcales }\end{array}$ & 2 & 6 & $2,06(, 29)$ & $2,19(, 63)$ & $-1,62(, 107)$ \\
\hline $\begin{array}{l}\text { Violencia asociada a mitos amor } \\
\text { romántico }\end{array}$ & 5 & 15 & $5,97(1,4)$ & $5,61(1,54)$ & $1,43(, 153)$ \\
\hline TOTAL Escala Ciberagresión & 23 & 69 & $25,34(3,33)$ & $25,81(5,22)$ & $-6,31(, 529)$ \\
\hline
\end{tabular}


Tabla 4

Contraste por sexo en las puntuaciones de la escala cibervictimización en cada una de sus dimensiones

\begin{tabular}{|c|c|c|c|c|c|}
\hline Dimensiones de la escala & $\begin{array}{l}\text { Mínima } \\
\text { teórica }\end{array}$ & $\begin{array}{l}\text { Máxim. } \\
\text { teórica }\end{array}$ & $\begin{array}{c}\text { Chicas } \\
\text { Mean (SD) }\end{array}$ & $\begin{array}{c}\text { Chicos } \\
\text { Mean (SD) }\end{array}$ & $\begin{array}{c}t \\
(p)\end{array}$ \\
\hline $\begin{array}{l}\text { Apartarse de la normatividad } \\
\text { sexual femenina }\end{array}$ & 5 & 15 & $5,45(, 1,05)$ & $5,30(, 97)$ & $8,84(, 37)$ \\
\hline $\begin{array}{l}\text { Transgredir } \\
\text { heteronormatividad sexual } \\
\text { obligatoria }\end{array}$ & 3 & 9 & $3,08(, 41)$ & $3,25(, 89)$ & $-1,44(, 15)$ \\
\hline $\begin{array}{l}\text { Imposiciones del canon de } \\
\text { belleza heteronormativo }\end{array}$ & 3 & 9 & $3,48(, 55)$ & $3,25(, 68)$ & $1,67(, 09)$ \\
\hline Estereotipos (rol familiar) & 1 & 3 & $1,25(, 47)$ & $1,12(, 44)$ & $1,72(, 09)$ \\
\hline Violencia sexual & 4 & 12 & $4,31(, 87)$ & $4,28(1,03)$ &, $16(, 87)$ \\
\hline $\begin{array}{l}\text { Violencia por manifestar } \\
\text { posiciones antipatriarcales }\end{array}$ & 2 & 6 & $2,21(, 79)$ & $2,10(, 43)$ &, $97(, 33)$ \\
\hline $\begin{array}{l}\text { Violencia asociada a mitos } \\
\text { amor romántico }\end{array}$ & 5 & 15 & $6,07(1,94)$ & $5,43(1,38)$ & $2,23(, 027)$ \\
\hline $\begin{array}{l}\text { TOTAL Escala } \\
\text { Cibervictimización }\end{array}$ & 23 & 69 & $25,86(5,05)$ & $24,75(4,48)$ & $1,36(, 17)$ \\
\hline
\end{tabular}

Tabla 5

Contraste por sexo en las puntuaciones de la escala ciberobservación en cada una de sus dimensiones

\begin{tabular}{|c|c|c|c|c|c|}
\hline Dimensiones de la escala & $\begin{array}{l}\text { Mínima } \\
\text { teórica }\end{array}$ & $\begin{array}{l}\text { Máxim. } \\
\text { teórica }\end{array}$ & $\begin{array}{c}\text { Chicas } \\
\text { Mean (SD) }\end{array}$ & $\begin{array}{c}\text { Chicos } \\
\text { Mean (SD) }\end{array}$ & $\begin{array}{c}t \\
(p)\end{array}$ \\
\hline $\begin{array}{l}\text { Apartarse de la normatividad } \\
\text { sexual femenina }\end{array}$ & 5 & 15 & $8,54(2,96)$ & $7,27(2,53)$ & $2,67(, 008)$ \\
\hline $\begin{array}{l}\text { Transgredir } \\
\text { heteronormatividad sexual } \\
\text { obligatoria }\end{array}$ & 3 & 9 & $4,62(1,82)$ & $4,33(1,71)$ &, $97(, 335)$ \\
\hline $\begin{array}{l}\text { Imposiciones del canon de } \\
\text { belleza heteronormativo }\end{array}$ & 3 & 9 & $5,32(2,03)$ & $4,27(1,62)$ & $3,35(, 001)$ \\
\hline Estereotipos (rol familiar) & 1 & 3 & $1,75(, 77)$ & $1,42(, 68)$ & $2,65(, 009)$ \\
\hline Violencia sexual & 4 & 12 & $6,18(2,29)$ & $5,28(2,04)$ & $2,43(, 016)$ \\
\hline $\begin{array}{l}\text { Violencia por manifestar } \\
\text { posiciones antipatriarcales }\end{array}$ & 2 & 6 & $3,04(1,30)$ & $2,63(1,06)$ & $2,05(, 042)$ \\
\hline $\begin{array}{l}\text { Violencia asociada a mitos } \\
\text { amor romántico }\end{array}$ & 5 & 15 & $8,94(3,14)$ & $6,91(2,73)$ & $4,04(, 000)$ \\
\hline $\begin{array}{l}\text { TOTAL Escala } \\
\text { Ciberobservación }\end{array}$ & 23 & 69 & $38,39(12,53)$ & $32,10(9,05)$ & $3,12(, 002)$ \\
\hline
\end{tabular}




\section{Puntuaciones obtenidas en la Cyberbullying Offending Scale}

La Cyberbullying offending scale (Hinduja \& Patchin, 2010) consta de 9 items Likert 5 puntos (tabla 6). Los resultados obtenidos denotan que las y los jóvenes no manifiestan participar en gran medida de las conductas de ciberbulling que se plantean en la escala. En una escala del 1 al 5, las puntuaciones medias de las y los participantes no llegan al 1.5.

Tabla 6

Descriptivos de la Cyberbullying Offending Scale

\begin{tabular}{lrrr}
\hline Ítems & N & Media & DS \\
\hline He acosado a otra persona por internet o móvil & 155 & 1,40 &, 971 \\
He colgado en internet comentarios crueles o hirientes sobre alguien & 155 & 1,41 &, 835 \\
He difundido rumores sobre alguien en internet & 155 & 1,22 &, 608 \\
He amenazado con hacer daño a alguien en internet & 155 & 1,30 &, 734 \\
He amenazado con hacer daño a alguien en un mensaje de móvil & 155 & 1,23 &, 625 \\
Me he hecho pasar por alguien y he actuado de forma cruel o hiriente & 155 & 1,23 &, 590 \\
He colgado una imagen cruel o hiriente de otra persona & 155 & 1,15 &, 498 \\
He creado una página web cruel o hiriente sobre alguien & 155 & 1,07 &, 379 \\
He colgado un vídeo cruel o hiriente de alguien & 155 & 1,09 &, 514 \\
\hline
\end{tabular}

Podemos identificar que las conductas que menos han puntuado están relacionadas con violencia mediante imagen, video o la creación de web. En cambio, obtienen unas puntuaciones ligeramente superiores las conductas ciberviolentas relacionadas con el acoso por móvil o internet, y realizar comentarios crueles por internet.

\section{Factores asociados a la ciberagresión en VG 2.0}

Los factores asociados con el comportamiento agresor se identificaron a partir de un análisis descriptivo de las variables de los casos con puntuaciones más altas en la escala de ciberagresión en VG 20. Y, en un segundo momento, mediante un análisis de regresión lineal múltiple (con el total de la misma escala como variable dependiente).

Dado que el comportamiento agresor es manifestado en un bajo porcentaje por la muestra del estudio, se procedió bajo el criterio empírico a seleccionar el percentil 70 de las puntuaciones del alumnado en la Escala de ciberagresión en VG2.0 (es decir los y las chicas que puntúan más alto en esta escala). El análisis descriptivo se realizó en base a todas las variables del cuestionario, resultando significativas las siguientes, que estarían asociadas con el comportamiento agresor (tabla 7): utilizar Twiter y Ask. fm de forma importante; puntuar más alto en la escala de Cyberbullying offending; puntuar más alto en todas las dimensiones de la escala de experiencias como agressor; ser de mayor edad y de cuarto curso; y tener pareja. No aparece significativo el sexo. 
Tabla 7

Descriptivos de las variables asociadas con la ciberagresión

\begin{tabular}{|c|c|c|c|c|c|c|}
\hline \multirow{2}{*}{\multicolumn{2}{|c|}{$\begin{array}{l}\text { Variables asociadas con el } \\
\text { comportamiento agresor }\end{array}$}} & \multirow{2}{*}{$\mathbf{n}$} & \multicolumn{4}{|c|}{ Variables continuas } \\
\hline & & & Media & Desviac & & p \\
\hline \multirow{4}{*}{ 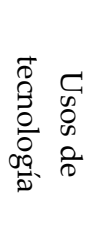 } & \multirow[t]{2}{*}{ Twitter } & 47 & \multicolumn{2}{|c|}{ Agresor $(1,65)$} & 78 & 031 \\
\hline & & 108 & \multicolumn{2}{|c|}{$\begin{array}{r}\text { No agressor } \\
(1,39)\end{array}$} & 64 & \\
\hline & \multirow[t]{2}{*}{ Ask.fm } & 47 & \multirow{2}{*}{\multicolumn{2}{|c|}{$\begin{array}{r}\text { Agresor }(1,91) \\
\text { No agressor } \\
(1,54)\end{array}$}} & \multirow{2}{*}{$\begin{array}{l}, 82 \\
, 76\end{array}$} & \multirow[t]{2}{*}{,008 } \\
\hline & & 108 & & & & \\
\hline \multirow{2}{*}{\multicolumn{2}{|c|}{ Escala Cyberbulling Offending }} & 47 & \multirow{2}{*}{\multicolumn{2}{|c|}{$\begin{array}{r}\text { Agresor }(1,57) \\
\text { No agressor } \\
(1,09)\end{array}$}} & \multirow{2}{*}{$\begin{array}{l}, 67 \\
, 20\end{array}$} & \multirow[t]{2}{*}{,000 } \\
\hline & & 108 & & & & \\
\hline \multirow{12}{*}{ 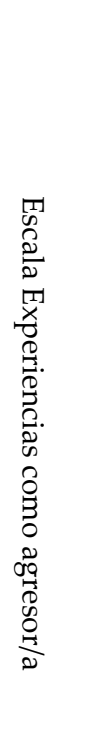 } & & 47 & \multirow{2}{*}{\multicolumn{2}{|c|}{$\begin{array}{r}\text { Agresor }(6,40) \\
\text { No agressor } \\
(5,09)\end{array}$}} & \multirow{2}{*}{$\begin{array}{r}1,58 \\
, 34\end{array}$} & \multirow[t]{2}{*}{,000 } \\
\hline & $\begin{array}{l}\text { normatividad sexual } \\
\text { femenina }\end{array}$ & 108 & & & & \\
\hline & Transgredir & 47 & \multicolumn{2}{|c|}{ Agresor $(3,70)$} & \multirow{2}{*}{$\begin{array}{l}, 93 \\
, 09\end{array}$} & \multirow[t]{2}{*}{,000 } \\
\hline & $\begin{array}{l}\text { heteronormatividad sexual } \\
\text { obligatoria }\end{array}$ & 108 & \multicolumn{2}{|c|}{$\begin{array}{r}\text { No agressor } \\
(3,00)\end{array}$} & & \\
\hline & Imposiciones del canon de & 47 & \multicolumn{2}{|c|}{ Agresor $(4,17)$} & 1,18 & \multirow[t]{2}{*}{,000 } \\
\hline & belleza heteronormativo & 108 & \multicolumn{2}{|c|}{$\begin{array}{r}\text { No agressor } \\
(3,15)\end{array}$} &, 41 & \\
\hline & \multirow[t]{2}{*}{ Estereotipos (rol familiar) } & 47 & Agres & $(1,48)$ & \multirow{2}{*}{$\begin{array}{l}, 62 \\
, 13\end{array}$} &, 000 \\
\hline & & 108 & No & $\begin{array}{r}\text { ressor } \\
(1,01)\end{array}$ & & \\
\hline & Violencia sexual & 47 & Agres & $(4,80)$ & 1,39 & ,000 \\
\hline & & 108 & No & $\begin{array}{r}\text { ressor } \\
(4,00)\end{array}$ & ,000 & \\
\hline & Violencia por manifestar & 47 & Agres & $(2,34)$ & ,78 & ,000 \\
\hline & posiciones antipatriarcales & 108 & No & $\begin{array}{r}\text { ressor } \\
(2,01)\end{array}$ & 13 & \\
\hline & Violencia asociada a mitos & 47 & Agres & $(7,25)$ & 1,97 & ,000 \\
\hline & amor romántico & 108 & No & $\begin{array}{r}\text { ressor } \\
(5,16)\end{array}$ &, 44 & \\
\hline Edad & & 47 & Agresc & $(14,82)$ & 1,68 & ,017 \\
\hline & & 108 & No & $\begin{array}{l}\text { ressor } \\
(14.09)\end{array}$ & 1,70 & \\
\hline & & & & riables c & górica & \\
\hline & & $\mathbf{n}$ & & $\%$ & & $\mathrm{p}$ \\
\hline Curso & & $\begin{array}{l}47 \\
108\end{array}$ & $\begin{array}{l}20,8 \% \text { de } \\
\text { de cuart }\end{array}$ & rimero y & $7 \%$ & 010 \\
\hline Tener & areja & $\begin{array}{l}47 \\
108\end{array}$ & $\begin{array}{l}45,5 \% \text { qu } \\
\text { que no }\end{array}$ & tienen y & $3 \%$ & ,010 \\
\hline
\end{tabular}


En el análisis de regresión lineal múltiple mediante el método de introducción, se consideró como variable dependiente la conducta ciberagresora (medida mediante la Escala de ciberagresión en VG2.0). Como variables independientes se consideraron en el análisis los usos de tecnologías, la competencia digital, la escala de ciberagresores de Hinduja \& Patchin (2010), el sexo, la edad, el tener pareja y la percepción de impunidad en la red.

Tabla 8

Análisis de regresión lineal múltiple en el comportamiento de ciberagresión en VG2.0

\begin{tabular}{|c|c|c|c|c|c|}
\hline \multirow{2}{*}{$\begin{array}{l}\text { Variable dependiente: conducta } \\
\text { ciberagresora }\end{array}$} & \multirow{2}{*}{$\begin{array}{c}\text { Coeficiente } \\
\text { Beta } \\
\text { estandarizado } \\
\end{array}$} & \multirow[b]{2}{*}{$\mathbf{t}$} & \multirow[b]{2}{*}{ Sig. } & \multicolumn{2}{|c|}{ Colinealidad } \\
\hline & & & & Tolerance & VIF \\
\hline (Constant) & & 13,675 & ,000 & & \\
\hline ¿Actualmente tienes pareja? & ,101 & 1,551 & ,123 & ,892 & 1,121 \\
\hline $\begin{array}{l}\text { ¿Sabes denunciar las fotografías subidas } \\
\text { a la red indebidamente? }\end{array}$ &,- 170 & $-2,616$ & ,010 & ,896 & 1,116 \\
\hline $\begin{array}{l}\text { ¿Crees que se castiga a las personas } \\
\text { que hacen cosas malas o ilegales en los } \\
\text { entornos on-line? }\end{array}$ &,- 086 & $-1,360$ & ,176 & ,958 & 1,043 \\
\hline Sexo & ,086 & 1,252 & 213 & ,808 & 1,238 \\
\hline $\begin{array}{l}\text { De la siguiente lista, ¿qué recursos } \\
\text { utilizas con mayor frecuencia? [Youtube] }\end{array}$ &,- 190 & $-2,791$ & ,006 & 823 & 1,216 \\
\hline $\begin{array}{l}\text { De la siguiente lista, ¿qué recursos } \\
\text { utilizas con mayor frecuencia? [Skype] }\end{array}$ & ,156 & 2,291 & 024 & 824 & 1,214 \\
\hline $\begin{array}{l}\text { De la siguiente lista, ¿qué recursos } \\
\text { utilizas con mayor frecuencia? [Ask.fm] }\end{array}$ & 168 & 2,578 & 011 & 893 & 1,120 \\
\hline Escala Cyberbulling & ,603 & 9,131 & ,000 & 872 & 1,147 \\
\hline
\end{tabular}

Los resultados evidencian un modelo de regresión válido con un $48 \%$ de predicción $\left(r^{2}=0.478\right)$. Se asumen los supuestos de linealidad, normalidad, independencia, y homocedasticidad. Las pruebas de colinealidad (incluidas en la tabla 8) identifican que no existe relación lineal exacta entre ninguna de las variables independientes. El modelo contempla las siguientes variables predictoras de la conducta cibreagresora en VG2.0:

- Puntuar más alto en la escala de ciberagresores (Hinduja \& Patchin, 2010). Es la variable predictora con mayor peso e indica que los jóvenes que puntúan más alto en la escala de experiencia como agresores también puntúan alto en la escala de ciberagresores.

- No saber denunciar fotografías indebidas. Es la segunda variable predictora e indica que los jóvenes que puntúan más alto en la escala de experiencia como agresores presentan menos competencia en esta categoría. 
- No utilizar Youtube. Es la tercera variable predictora e indica que los jóvenes que puntúan más alto en la escala de experiencia como agresores utilizan en menor medida este entorno.

- Usar Ask.fm. Indica que los jóvenes que puntúan más alto en la escala de experiencia como agresores usan de forma importante este entorno.

- Usar Skype. Indica que los jóvenes que puntúan más alto en la escala de experiencia como agresores usan de forma importante este entorno.

\section{Discusión y conclusiones}

\section{Discusión}

El estudio ha dado respuesta a los dos objetivos planteados. Por un lado ha evidenciado las experiencias de las y los jóvenes con relación a la violencia de género 2.0. Las puntuaciones indican que todos los jóvenes de la muestra alguna vez han tenido experiencias de ciberagresión.

En la escala de ciberagresión los chicos puntúan más alto que las chicas, pero las diferencias no son estadísticamente significativas, excepto en las conductas violentas hacia gais, lesbianas y transexuales donde los chicos sobresalen. En consonancia con estos resultados en el reciente estudio de Durán y Martínez-Pecino (2015) sobre conductas de victimización y ciberacoso a través del teléfono móvil y de Internet en las relaciones de noviazgo en universitarios, los resultados concluyeron, en contra de lo esperado, que los chicos informaron de mayores niveles de victimización por parte de sus parejas que las chicas, a través de los dos medios, en la misma dirección de otros trabajos realizados con jóvenes en otros países (Burke et al., citado en Durán \& Martínez-Pecino, 2015). En lugar de estar eliminándose el nivel de conductas violentas perpetradas por los chicos, (García Fernández et al., 2015) las chicas se están sumando a este tipo de comportamiento. Sin embargo, las consecuencias de estos tipos de agresiones son más perjudiciales para las mujeres (Banyard \& Cross, 2008; Borrajo, Gámez-Guadix \& Calvete, 2015; Estébanez \& Vázquez, 2013; Torres, 2013), entre otras razones por su posición de vulnerabilidad psicológica en el entramado relacional y social. Deberían seguirse estudiando el papel del sexo en las ciberagresiones (Borrajo et al., 2015) y analizar si son reactivas o proactivas y si las chicas utilizan las agresiones online como mecanismo de defensa o respuestas a agresiones anteriores.

Las diferencias estadísticas en conductas violentas hacia homosexuales por parte de los chicos apoya la hipótesis de que el género que encontramos en la red es heteronormativo, en el que mujeres y cualquier persona que se sitúe fuera de los márgenes de lo "patriarcalmente" aceptable", se convierte en un colectivo vulnerable de ser agredido o acosado a través de la red.

En la escala de cibervictimización puntúan más alto chicas que chicos, pero las diferencias estadísticas solo se dan en la dimensión mitos del amor romántico, donde las chicas padecen más las ciberagresiones asociadas a esta dimensión. Las creencias del amor romántico que asigna a las mujeres un lugar en las relaciones afectivas y los mecanismos legitimados por el patriarcado en la relación amorosa como el control, 
la posesión y los celos, (Esteban \& Távora, 2008; Esteban, 2011; Ferrer \& Bosch, 2013) están en la base de este tipo de conductas abusivas. Conductas además ampliamente normalizadas en la juventud (Borrajo et al., 2015). Este tipo de violencia aparece en un período de inestabilidad personal, en que las jóvenes se encuentran en un proceso de construcción identitaria. Los episodios violentos que sufren las chicas pueden afectar de manera traumática la formación psíquica, cognitiva y relacional de la persona, en un período de alta vulnerabilidad, en el que las relaciones amorosas constituyen un elemento central para su desarrollo social y afectivo (Vézina, 2012).

La escala de ciberobservación es la que obtiene la puntuación más alta. Es incongruente que hayan sido más observadores que agresores o víctimas, probablemente cuesta reconocerse como persona víctima o como agresora, en cambio es más fácil reconocerse como observador. En la investigación de Garmendia, Garitaonandia, Martínez y Casado (2011), el porcentaje de jóvenes que habían sido víctimas de acoso siempre estaba por encima de los que los habían acosado.

Hay diferencias significativas en la escala de ciberobservación entre chicos y chicas. Posiblemente las chicas tienen más sensibilidad y conciencia a las violencias de género.

Con relación al segundo objetivo de la investigación, se han encontrado unos factores asociados al comportamiento ciberagresor, para cuya discusión nos centraremos en el uso de los entornos tecnológicos y en la correlación alta que obtiene el perfil de agresor en la escala de ciberagresores de Hinduja y Patchin, (2010).

Los entornos nos hacen cuestionarnos sobre la utilización de entornos activos como ask y Skype, los más utilizados, y un entorno pasivo como youtube, el menos utilizado.

Ask es la red social que seduce a los jóvenes porque permite hacer preguntas de forma anónima, ahí es donde reside la clave de su éxito. Skype por su parte es un entorno de conversación, tanto con gente conocida como desconocida que también permite la obtención de datos privados. A menudo se utiliza como entorno secundario una vez se ha contactado primero por otros medios y es muy usado en los juegos online. No es de extrañar que las características de estos entornos sean más valoradas por las persones agresoras, pues les permiten dar más rienda suelta a sus actos.

El otro factor a considerar dentro del perfil de ciberagresión es la correlación con las puntuaciones obtenidas en la escala de ciberagresores de Hinduja y Patchin, (2010). Las agresiones en función del género correlacionan con agresiones de ciberbullyng en general. Las actitudes agresoras marcan una pauta que es generalizable a cualquier conducta.

\section{Conclusiones}

Esta investigación pone a disposición de la comunidad académica un instrumento para analizar las agresiones que se producen en espacios virtuales motivadas por discriminaciones en función del género. A nivel internacional no hemos encontrado otro instrumento de estas características. Los estudios que contemplan la dimensión género suelen ser cualitativos y estar centrados en agresiones producidas por parejas o exparejas. No hay constancia de ningún instrumento que vaya más allá de las ciberagresiones en relaciones afectivas-sexuales. Los índices de fiabilidad son altos y se ha podido establecer la validez ligada al criterio. 
Este trabajo ofrece además una clasificación de conductas exhibidas en la red para estudiar el fenómeno de las agresiones en función del género. Se toma como marco explicativo de la clasificación y las conductas el sistema patriarcal. Un sistema que normativiza las conductas y los roles y castiga a quienes se apartan de esta normatividad.

Los resultados marcan la tendencia, ya apuntada, del fenómeno de la ciberagresión, cada vez con índices más altos y que debe preocupar a responsables políticos y educativos.

El debate de la asimetría o simetría entre chicos y chicas en relación a la violencia continúa, pero queremos remarcar la deportación de las chicas al comportamiento masculino como una tendencia peligrosa y que plantea grandes interrogantes a la acción educativa.

Queremos remarcar la importancia de programas preventivos sobre las relaciones amorosas. Las creencias distorsionadas que tienen la juventud sobre el amor justifican conductas abusivas de control, dominio y ejercicio de la violencia especialmente hacia las chicas por parte de los chicos. Algo que ha quedado demostrado en nuestra muestra.

Finalmente apuntamos la limitación del trabajo con relación a la muestra que deberá ampliarse en futuros estudios. Los resultados deben interpretarse, por tanto, en clave de significatividad y no de generalización.

Igualmente deberá ampliarse el ámbito geográfico de la muestra y la edad estudiada. En cuando a la franja de edad estudiada, sería interesante ver cómo funciona el instrumento con otras edades por encima o por debajo del rango que se presenta.

\section{Referencias}

Alexy, E.M., Burgess, A. W., Baker, T., \& Smoyak, S. A. (2005). Perceptions of Cyberstalking Among College Students. Brief Treatment and Crisis Intervention, 5(3), 279- 289. doi: 10.1093/brief treatment/mhi020.

Arisó, O. \& Mérida, R. (2010). Los géneros de la violencia Una reflexión queer sobre la violencia de género. Barcelona: Egales.

Banyard, V. L., \& Cross, C. (2008). Consequences of teen dating violence understanding intervening variables in ecological context. Violence Against Women, 14(9), 998-1013.

Barlett, C., \& Coyne, S. M. (2014). A meta-analysis of sex differences in cyber-bullying behavior: The moderating role of age. Aggressive Behavior, 40(5), 474-488.

Beckman, L., Hagquist, C., \& Hellström, L. (2013). Discrepant gender patterns for cyberbullying and traditional bullying. An analysis of Swedish adolescent data. Computers in Human Behavior, 29(5), 1896-1903. doi:10.1016/j.chb.2013.03.010.

Borrajo, E., Gámez-Guadix, M., \& Calvete, E. (2015). Justification beliefs of violence, myths about love and cyber dating abuse. Psicothema, 27(4), 327-333. doi: 10.7334/ psicothema2015.59.

Buelga, S., \& Pons, J. (2012) Agresiones entre Adolescentes a través del Teléfono Móvil y de Internet. Psychosocial Intervention, 21(1), 91-101.

Burke, S.C., Wallen, M., Vail-Smith, K., \& Knox, D. (2011). Using technology to control intimate partners: An exploratory study of college undergraduates. Computers in Human Behavior, 27(3), 1162-1167. doi: 10.1016/j.chb.2010.12.010. 
Calmaestra, J. (2011). Cyberbullying: prevalencia y características de un nuevo tipo de bullying indirecto. (Tesis doctoral). Servicio de Publicaciones de la Universidad de Córdoba, Córdoba.

Calvete, E., Orue, I., Estévez, A., Villardón, L., \& Padilla, P. (2010). Cyberbullying in adolescents: Modalities and aggressors profile. Computers in Human Behavior, 26, 1128-1135. doi:10.1016/j.chb.2010.03.017.

Defensor Del Pueblo-UNICEF (2006). Violencia escolar: el maltrato entre iguales en la educación secundaria obligatoria 1999-2006. Madrid: Publicaciones de la Oficina del Defensor del Pueblo.

Dimond, J. P., Fiesler, C., \& Bruckman, A. S. (2011). Domestic violence and information communication technologies. Interacting with Computers, 23(5), 413-421.

Donoso-Vázquez, T., Rubio, M.J., Velasco, A., \& Vilà, R. (2014). Cuestionario de violencias de género 2.0. Recuperado de http://hdl.handle.net/2445/53384.

Donoso-Vázquez, T., Rubio, M.J., Vilà, R., Velasco, A., \& Aneas, A. (2014). La medición del acoso en Internet. Comunicación presentada en II Congreso Internacional de Ciencias de la Educación y del Desarrollo. Granada, España.

Durán, M. \& Martínez-Pecino, R. (2015). Ciberacoso mediante teléfono móvil e Internet en las relaciones de noviazgo entre jóvenes. Comunicar, XXII, 44, 159-167.

Esteban, M.L., \& Távora; A. (2008). El amor romántico y la subordinación social de las mujeres: revisiones y propuestas. Anuario de psicología, 39(1), 59-73.

Esteban, M.L. (2011). Crítica del Pensamiento Amoroso. Barcelona: Bellaterra.

Estébanez, I., \& Vázquez, N. (2013). La desigualdad de género y el sexismo en las redes sociales. Bilbao: Observatorio vasco de la Juventud.

Ferrer, V., \& Bosch, E. (2013). Del amor romántico a la violencia de género. Para una coeducación emocional en la agenda educativa. Profesorado. Revista de curriculum y formación del profesorado, 17(1), 105-122.

Finn, J., \& Atkinson, T. (2009). Promoting the safe and strategic use of technology for victims of intimate partner violence: Evaluation of the Technology Safety Project. Journal of Family Violence, 24(1), 53-59. doi: 10.1007/s10896-008-9207-2

García Fernández, C.M, Romera, E.M., \& Ortega Ruiz, R. (2015). Explicative factors of face-to-face harassment and cyberbullying in a sample of primary students. Psicothema, 27(4), 347-353. doi: 10.7334/psicothema2015.35

Garmendia, M., Garitaonandia, C., Martínez, G., \& Casado, M.A. (2011). EU Kids Online II: Mejorando el conocimiento sobre el uso y la seguridad en Internet de los menores en Europa. Recuperado de www.ehu.es/eukidsonline

Hinduja S., \& Patchin J.W. (2008). Cyberbullying: an exploratory analysis of factors related to offending and victimization, Deviant Behavior, 29(2), 129-156. doi:10.1080/01639620701457816.

Hinduja S., \& Patchin J.W. (2010). Bullying, Cyberbullying, and Suicide. Archives of Suicide Research, 14(3), 206-221.

Hyrkäs, K., Appelqvist-Schmidlechner, K., \& Oksa, L. (2003). Validating an instrument for clinical supervision using an expert panel. International Journal of nursing studies, $40(6), 619-625$.

Li, Q. (2007). New bottle but old wine: a research of cyberbullying in schools. Computers in Human Behavior, 23(4), 1777-1791. doi:10.1016/j.chb. 2005.10.005. 
Modecki, K. L., Minchin, J., Harbaugh, A. G., Guerra, N. G., \& Runions, K. C. (2014). Bullying prevalence across contexts: a meta-analysis measuring cyber and traditional bullying. Journal of Adolescent Health, 55(5), 602-611.

Mora-Merchán, J. A., Ortega, R., Justicia, F., \& Benítez, J. L. (2001). Violencia entre iguales en escuelas andaluzas. Un estudio exploratorio utilizando el cuestionario general europeo TMR. Revista de Educación, 325, 323-338.

Muñoz-Rivas, M. J., Graña Gómez, J. L., O’Leary, K. D., \& González Lozano, P. (2007). Aggression in adolescent dating relationships: prevalence, justification, and health consequences. Journal of Adolescent Health, 40(4), 298-304.

Nunnally, J. C. (1978). Psychometric Theory. New York: McGraw-Hill.

Ortega, R., Calmaestra, J., \& Mora-Merchán, J. A. (2008). Cyberbullying. International Journal of Psychology and Psychological Therapy, 8(2), 183-192.

Skjong, R., \& Wentworth, B. (2000). Expert Judgement and risk perception. Recuperado de http://research.dnv.com/skj/Papers/SkjWen.pdf.

Smith, P.K. (2006). Ciberacoso: naturaleza y extensión de un nuevo tipo de acoso dentro y fuera de la escuela. Recuperado de: http://www.observatorioperu.com/lecturas/ciberacoso_pSmith.pdf.

Southworth, C., Dawson, S., Fraser, C., \& Tucker, S. (2005). A high-tech twist on abuse: Technology, intimate partners talking, and advocacy. Violence Against Women. Online. Recuperado de: http://nnedv.org/downloads/SafetyNet/NNEDV_HighTechTwist_PaperAndApxA_English08.pdf

Spence-Diehl, E. (2003). Stalking and technology: The double-edged sword. Journal of Technology and Human Services, 22(1), 5-18. doi: org/10.1300/J017v22n01_02

Torres, C. (2013). El ciberacoso como forma de ejercer la violencia de género en la juventud: un riesgo en la sociedad de la información y del conocimiento. Madrid: Ministerio de sanid, servicios sociales e igualdad. Recuperado de: https://www.poder-judicial.go.cr/ observatoriodegenero/wp-content/uploads/2016/06/Ciberacoso-como-violencia-degenero.pdf.

Vézina, J. (2012). La violence subie dans les relations amoureuses des filles à l'adolescence et au début de l'âge adulte: style de vie à risque et contextes associés. Thèse presenté com a exigence partielle du doctoral en psychologie. (Tesis doctotal, Université de Québec à Montréal). Recuperado de http://www.archipel.uqam.ca/4603/

Fecha de recepción: 6 de febrero de 2016

Fecha de revisión: 6 de febrero de 2016

Fecha de aceptación: 18 de octubre de 2016 